\title{
A focused telephonic nursing intervention delivers improved adherence to A1c testing
}

Patty M. Orr

Healthways, Inc.

Matthew A. McGinnis

Healthways, Inc.

Laurel R. Hudson

Healthways, Inc.

Sadie S. Coberley

Healthways, Inc.

Alhert frisa uafarddditional works at: https://jdc.jefferson.edu/healthpolicyfaculty Thomas Jefferson University

Part of the Health Services Research Commons

\section{Let us know how access to this document benefits you}

\section{Recommended Citation}

Orr, Patty M. ; McGinnis, Matthew A.; Hudson, Laurel R.; Coberley, Sadie S.; Crawford, Albert; Clarke, Janice L.; and Goldfarb, Neil I., "A focused telephonic nursing intervention delivers improved adherence to A1c testing" (2006). College of Population Health Faculty Papers. Paper 30.

https://jdc.jefferson.edu/healthpolicyfaculty/30

This Article is brought to you for free and open access by the Jefferson Digital Commons. The Jefferson Digital Commons is a service of Thomas Jefferson University's Center for Teaching and Learning (CTL). The Commons is a showcase for Jefferson books and journals, peer-reviewed scholarly publications, unique historical collections from the University archives, and teaching tools. The Jefferson Digital Commons allows researchers and interested readers anywhere in the world to learn about and keep up to date with Jefferson scholarship. This article has been accepted for inclusion in College of Population Health Faculty Papers by an authorized administrator of the Jefferson Digital Commons. For more information, please contact: JeffersonDigitalCommons@jefferson.edu. 


\section{Authors}

Patty M. Orr, Matthew A. McGinnis, Laurel R. Hudson, Sadie S. Coberley, Albert Crawford, Janice L. Clarke, and Neil I. Goldfarb 


\title{
A Focused Telephonic Nursing Intervention Delivers Improved Adherence to A1c Testing
}

\author{
PATTY M. ORR, M.S.N., Ed.D., R.N., ${ }^{1}$ MATTHEW A. McGINNIS, B.S., ${ }^{1}$ \\ LAUREL R. HUDSON, M.S.N., R.N., ${ }^{1}$ SADIE S. COBERLEY, Ph.D., ${ }^{1}$ \\ ALBERT CRAWFORD, Ph.D., M.B.A., M.S.I.S., ${ }^{2}$ JANICE L. CLARKE, R.N., ${ }^{2}$ \\ and NEIL I. GOLDFARB ${ }^{2}$
}

\begin{abstract}
Compliance with hemoglobin A1c (A1c) testing is suboptimal despite the clear national recommendations and guidelines established for care of patients with diabetes. Recent studies have demonstrated a relationship between participation in a diabetes disease management (DM) program and improved adherence to A1c testing. A focused intervention study was initiated to investigate the ability of a DM program to drive improvement in A1c testing. A cohort of 36,327 members experienced a statistically significant increase (29\%) in A1c testing while participating in the 6-month focused intervention. This finding demonstrated that a focused DM intervention is able to deliver improvement in a clinical process metric critical for managing patients with diabetes, thereby reducing their risk of disease exacerbation. (Disease Management 2006;9:277-283)
\end{abstract}

\section{INTRODUCTION}

D IABETES imposes an enormous health burden on the United States every year. In 2002, 18.2 million Americans had diabetes (6.3\% of the population), and the associated costs totaled $\$ 132$ billion ( $\$ 92$ billion direct; $\$ 40$ billion indirect). ${ }^{1}$ Nationwide efforts are under way to delay the progression of diabetes and its complications, thereby reducing these tremendous costs. Routine hemoglobin A1c (A1c) testing is an essential component of the initiatives aimed at reduction of short- and long-term complications of diabetes.

The medical literature is replete with evidence that improving glycemic control creates benefits for persons with diabetes by lowering diabetes-related costs and decreasing shortand long-term diabetes complications. ${ }^{2-5}$ The Diabetes Control and Complications Trial (DCCT) found that persons receiving intensive insulin treatment to reach a glycemic goal of $6.05 \%$ or less had dramatic relative reductions in risk for microvascular and neurological end points, including greater than $70 \%$ reduction for clinically important sustained retinopathy, $56 \%$ for laser photocoagulation, 39\% for microalbuminuria, and $60 \%$ for confirmed clinical neuropathy. Although they did not reach statistical significance, specific macrovascular end points realized an approximate $41 \%$ risk reduction for combined macrovascular events. ${ }^{5}$

\footnotetext{
${ }^{1}$ Healthways, Inc, Nashville, Tennessee.

${ }^{2}$ Department of Health Policy, Jefferson Medical College, Philadelphia, Pennsylvania.
} 
In an alternate study, it was demonstrated that, for a person with an A1c value of $6 \%$, successive $1 \%$ increases in A1c resulted in medical expense increases of approximately $4 \%, 10 \%$, $20 \%$, and $30 \%$. Moreover, increases in medical charges for defined inpatient and outpatient services accelerated as A1c values increased. ${ }^{6}$

Recognizing the importance of regularly scheduled A1c testing in the control of diabetes, the American Diabetes Association (ADA) and the American College of Endocrinology established guidelines regarding frequency of A1c testing. The American College of Endocrinology/American Association of Clinical Endocrinologists (ACE/AACE) recommends that an A1c test be performed during an initial assessment and during follow-up assessments, which should occur at no longer than 3-month intervals. The ACE/AACE also recommends that $\mathrm{A} 1 \mathrm{c}$ be universally adopted as the primary method of assessment of glycemic control. The ACE/AACE target for glycemic control is an A1c value less than or equal to $6.5 \% .^{7}$ Similarly, the ADA recommends obtaining an A1c during the initial assessment and then routinely as part of continuing care. The ADA also recommends that $\mathrm{A} 1 \mathrm{c}$ be obtained at least twice a year in patients who are meeting treatment goals and whose glycemic control is stable. More frequent testing (ie, quarterly assessment) is recommended for patients whose therapy has been changed and for patients who are not meeting treatment and glycemic goals. The $\mathrm{ADA}^{\prime}$ 's target $\mathrm{A} 1 \mathrm{c}$ is less than $7 \%{ }^{8}$

In general, adherence to diabetes standards of care, such as adherence to A1c testing frequency guidelines, is less than optimal. ${ }^{8,9}$ Disease management (DM) programs have a unique opportunity to provide a solution. As a chronic, complex condition affecting large populations, diabetes lends itself to DM strategies. In fact, diabetes is the most frequently included condition among DM program offerings. While numerous studies have demonstrated the ability of DM to decrease A1c levels, ${ }^{3,10}$ there are few analyses of the impact of $\mathrm{DM}$ on the frequency of A1c testing in a large population.

Performance of an A1c test at the recommended frequency is essential for establishing goals to lower A1c levels, thereby improving diabetes outcomes. Results of recent Healthways, Inc. (Nashville, TN) studies of the impact of interventions on adherence to A1c testing showed an association between patient participation in the diabetes DM program and a measurable improvement in adherence. ${ }^{11}$ The purpose of this study was to determine the ability of a focused telephonic intervention to directly drive improvement in patient adherence to recommended A1c testing.

\section{METHODS}

\section{Diabetes disease management program overview}

DM programs were conceived as a means to work proactively with persons with chronic conditions, via telephone and mail, to ensure that their health and wellness needs are met. DM telephone communications focus on improving each member's understanding of his or her condition, and fostering adherence to standards of care and treatment regimens. The seven call centers in this study were staffed with clinicians (ie, Registered Nurses and Registered Dieticians) who initiated and maintained telephonic relationships with DM program members. Enrollment in this program was via an "opt-out" process (ie, persons with diabetes were automatically enrolled in the DM program with the right to decline participation). For a member under the age of 18, the telephone call was received by a parent or legal guardian unless the member had parental permission to receive the call directly. Similarly, caregivers could receive telephone calls on behalf of elderly or disabled members with the member's approval

Telephonic member support included several types of phone calls, among them introductory welcome calls, regularly scheduled care calls based on level of disease severity, and reminder calls regarding standards of care. Mail interventions included quarterly newsletters, reminder mailings, and disease-specific educational materials. An introduction letter was mailed to both the patient and the corresponding physician. This was followed by a welcome packet to members with materials explaining the program in further detail. Once the 
telephonic intervention was implemented, additional materials were mailed to members as needed.

\section{Population identification}

From a diabetes DM population drawn from 16 different health plans, members with moderate-to-severe risk of diabetes complications were identified using a proprietary risk stratification methodology. In general terms, members were stratified based upon the severity and control of their diabetes, and their utilization of emergency department and hospital resources. Eligibility criteria included (1) callability (ie, reachable by telephone), (2) enrollment in the DM program for the continuous 12month study period, and (3) stratification in the top half of the population relative to acuity. The 36,327 members who met the eligibility criteria represented approximately $19.7 \%$ of the diabetes DM population. The study population was $48.7 \%$ female, and the mean age was 57.29 years.

\section{The focused intervention}

When on a routine call with a member in the population identified above, the clinician reviewed the member's electronic DM records for evidence of an A1c test being performed in the previous 6 months. Evidence of an A1c test exists in the electronic DM record primarily as an administrative claim obtained from the health plan. However, in the absence of an administrative claim for the test, the clinician asked if the member had received an A1c test in the past 6 months. If the response was positive, the clinician documented this as a self-reported claim along with the date and results of the test in the member's electronic DM record.

If the member had not been tested during the past 6 months, the clinician initiated a conversation about the importance of A1c testing in diabetes management, including the recommended frequency of testing. Clinicians referred to a script to ensure consistency and completeness of the message conveyed to members. A specific goal, created with the member during the conversation (eg, "I will talk to my doctor about completing an A1c."), was subsequently mailed to the member's home address. After a 2-week interval, a focused follow-up phone call was made to check on the member's progress in meeting the goal of obtaining an A1c test. Focused phone calls were repeated every 2 weeks, integrating with regularly scheduled care calls when needed, until the A1c test had been completed or until the 6-month study period had ended.

\section{Data dollection and analysis}

The 6-month preintervention period was defined as September 1, 2003 through February 29,2004 . The 6-month focused intervention period included March 1, 2004 through August 31, 2004 (with a 4-month run-out to allow for the inclusion of late medical claims). A1c testing adherence was determined by evidence of a test being performed in the electronic DM record, represented primarily by administrative claims, and secondarily via self-report from the members. Two subsets of members were established: (1) those with administrative claims and (2) those with self-reported claims.

The electronic DM records for the identified population were evaluated for laboratory claims and self-reports of an A1c test in the preintervention and focused intervention periods. The member records were flagged for each period with " $\mathrm{Y}$ " (evidence of A1c) or "N" (no evidence of A1c). An adherence percentage was calculated for each period (ie, total number of members having an A1c test/total members in population). Additional analyses were performed to measure the A1c testing percentages for members with self-reported A1c tests compared to members with A1c tests documented by administrative claims.

\section{Statistical analysis}

The results were tested for statistical significance using a $Z$ test.

\section{RESULTS}

At the completion of the 6-month focused intervention, the aggregate number of members who received an A1c test increased 12\%, from 14,843 to 19,235 members (Fig. 1). This represented a statistically significant increase in A1c 


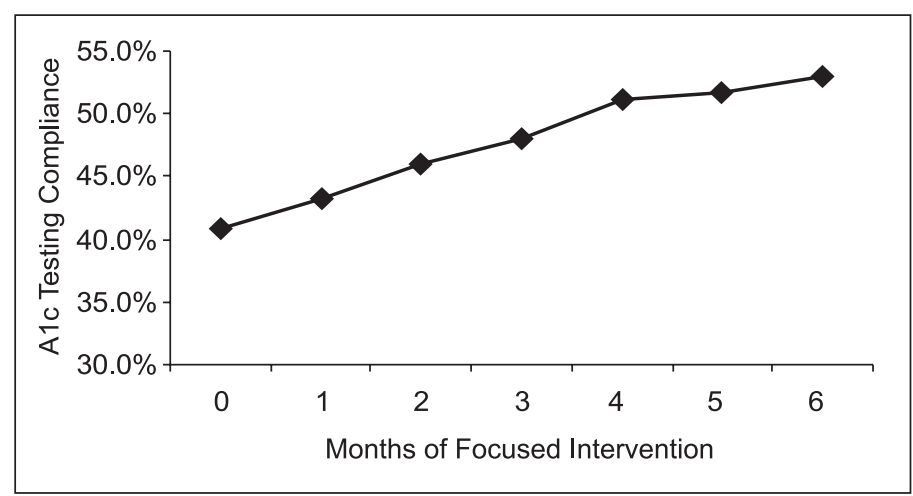

FIG. 1. Improvement in hemoglobin A1c (A1c) adherence across months of the focused intervention. Month zero shows A1c testing in the last month of the preintervention period.

testing adherence, from $40.9 \%$ to $52.9 \%(29 \%$ relative increase) for patients in the moderateto-severe risk strata for diabetes complications $(p<0.0001, Z=-32.651)$.

Gender appeared to have no impact on adherence to A1c testing. Females experienced a $12.4 \%$ improvement (from $41.3 \%$ to $53.8 \%$ ), whereas males had an $11.8 \%$ increase $(40.4 \%$ to $52.2 \%$ ) in A1c testing.

Age appeared to influence A1c testing adherence (Fig. 2). The greatest improvement was observed in the 0-19 age group; within this group, those aged 0-9 years had a testing increase of $14.9 \%$, and those aged $10-19$ years had an increase of $20.4 \%$. Members aged 50-69 experienced large increases as well; a $12.7 \%$ increase for those aged 50-59 years and a $13.4 \%$ increase for those aged 60-69 years. All other age categories demonstrated increases in A1C testing, ranging from $8.1 \%$ to $10.6 \%$.

Seventy-two percent of the A1c tests recorded in the electronic DM record were acquired from administrative claims data. Evaluation of testing adherence for members with self-reported A1c tests compared to members with A1c tests documented by administrative claims data revealed similar increases, 5.9\% and $6.6 \%$, respectively, after 6 months of focused intervention. This improvement in A1C testing adherence was statistically significant $(p<0.0001, Z=-24.632)$ for both member groups.

The call differential analysis (ie, the number of additional calls received by a member in the

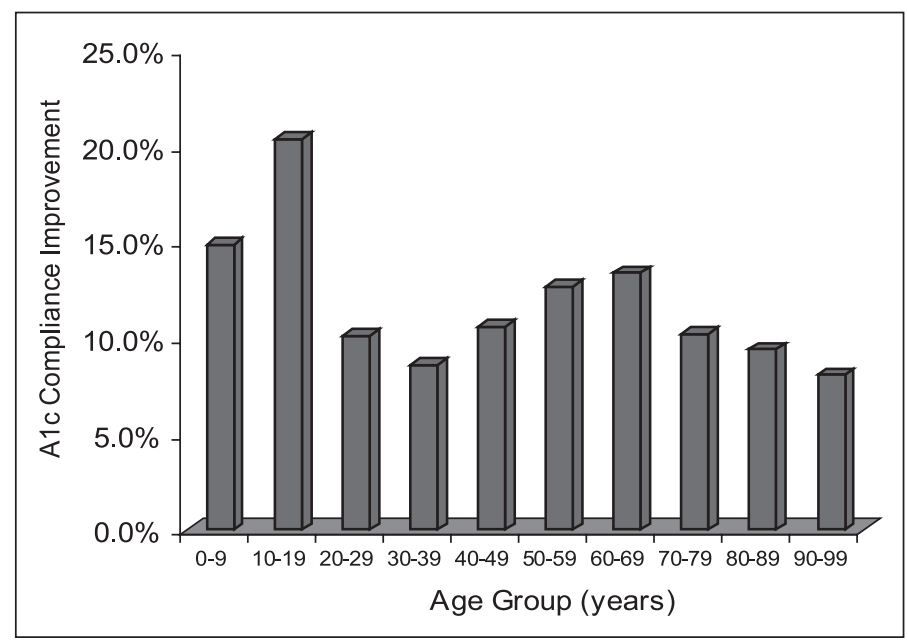

FIG. 2. Percent increase in hemoglobin A1c (A1c) testing adherence across age groups during the focused intervention period compared to the preintervention period. 
focused intervention period when compared to preintervention) revealed a positive correlation between incremental increases in the number of calls and the observed increases in testing adherence (Fig. 3). The addition of one, two, three, or four phone calls during the focused intervention led to corresponding increases in A1c testing adherence of $14.0 \%, 16.1 \%, 17.3 \%$, and $23.6 \%$, respectively, compared to the preintervention period.

\section{DISCUSSION}

The focused diabetes DM intervention was associated with significant increases in A1c testing adherence in a large population $(n=$ 36,327 ) over a 6 -month period. This finding suggests that a short-term telephonic intervention can positively impact patient adherence to a clinical process metric relevant to their diabetes disease control. The increase in A1c testing also demonstrates the potential for patient initiation to serve as an effective tool for positive change in physician performance on standard-of-care measures. Such results suggest that DM interventions can encourage patients to partner with their physicians in managing their conditions, thus strengthening the patient/physician relationship.

While no differences were observed with respect to gender in this study, adherence to A1c

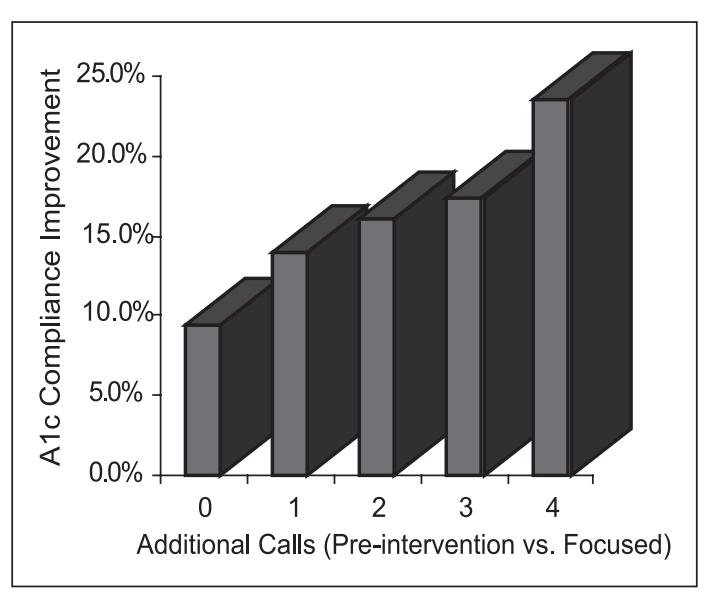

FIG. 3. Hemoglobin A1c (A1c) adherence improvement-call differential comparison. Members who received one to four more calls during the focused intervention period compared to the preintervention period had higher A1c testing adherence. testing appeared to be affected by patient age. Members aged 0-19 years demonstrated the best improvement (ie, testing compliance increased from $14.9 \%$ to $20.4 \%$ for this population). This may be explained by parental involvement and the assumption that parents of children with diabetes are particularly receptive to guidance that ensures the health and safety of their children. Specifically, members in the aged 10-19 subgroup demonstrated greater improvement in A1c testing compliance than the total group. This may stem from the members having parental permission to receive the intervention calls and to become more involved in managing their diabetes.

Members aged 50-69 years had the next largest improvement in A1c testing adherence: $12.7 \%$ of those aged $50-59$ years and $13.4 \%$ of those aged 60-69 years. While young enough to actively care for themselves, these members may be more likely to experience significant physical and health-related changes, which may motivate improvements in adherence.

Those members in the age groups 80-89, 90-99, and 30-39 demonstrated improvements of $9.4 \%, 8.1 \%$, and $8.6 \%$, respectively. Illness and frailty may hinder elderly members in taking the recommended action steps for their health. For those aged 30-39 years, factors related to employment and child care may interfere with scheduling and keeping additional healthcare appointments. Similarly, members in this age group may be less likely to be at home to receive focused intervention telephone calls.

Call differential proved to be an interesting end point in this study. The results suggest that increases in the number of phone calls and goal-setting conversations are associated with subsequent increases in A1c testing adherence. When compared to the standard number of calls received in the preintervention phase, members receiving one, two, three, or four additional phone calls during the intervention demonstrated steady increases in adherence as the number of calls and opportunities for goal setting increased. Longerterm studies in similar populations might reveal the exact nature of this relationship, and be useful for program design and business model improvements. 
In general, this study supports the hypothesis that improvement in clinical process metrics in a large population can be driven by a DM program, in this case by a focused telephonic nursing intervention. Evidence of the ability to impact enormous numbers of individuals in a relatively short time is encouraging, not just for the DM industry, but for the care of diabetes populations as a whole.

\section{Limitations}

Data collection. This research examined both administrative medical claims and self-reports to determine A1c testing adherence. Although select studies have indicated that persons with diabetes can accurately report whether or not they have received an A1c test in the past year, ${ }^{12}$ other studies indicate that self-reports of A1c tests overstate reality. ${ }^{13}$ Future studies might investigate the accuracy of self-reports by following A1c adherence in a group of members with administrative claims data for their ability to accurately report the performance of an A1c test.

Length of study period. To fully understand the effect of DM programs on A1c testing adherence, future studies might be designed to analyze the longer-term impact of such an intervention on a diabetes population. For instance, a longer study period might allow an examination of whether the intervention produced only short-term adherence (ie, members obtained one A1c test during the 6-month study period) or a sustained behavior change (ie, members continued to obtain tests as often as recommended). A longer study period also will be important in assessing the relationship between contact with members by telephone (ie, calls), setting goals, and driving improvement in outcomes.

Generalizability. While drawn from 16 different health plans in different regions of the United States, the population studied herein had diabetes severity scores ranging from moderate to severe according to a proprietary risk stratification model. Because of the higher burden of disease, these members may be more likely to be at home to receive the intervention phone calls. Specifically, their illness may interfere with their ability to be employed full- time or otherwise out of the home. A combination of unmeasured variables may influence the particular receptivity of this population to the intervention program, including the average adherence rate prior to the intervention (low vs. high), the type of metric under consideration, and the disease population of interest. The potential effects of these additional variables undermine the ability to extrapolate these results to a larger, more generic chronically ill population.

\section{Future research}

Future study designs might take into consideration the limitations noted above and will include:

- Randomizing the population into a focused intervention DM group and a standard DM control group for comparison

- Constructing analyses that examine the relationship between adherence to testing and outcomes (ie, clinical values, disease control, and costs)

- Lengthening the intervention duration to 12 or more months

\section{CONCLUSION}

To date, few studies have examined the potential of DM to improve clinical process metrics across large populations of chronically ill individuals. This study suggests that this focused DM intervention is associated with increased adherence to A1c testing guidelines in a large diabetes population. In addition, these findings suggest that, equipped with the appropriate knowledge and communication skills, patients can contribute toward improving their adherence to recognized standards of care. This study provides a foundation for a variety of similar studies to investigate the specific role of DM programs in empowering patients to improve their health.

\section{REFERENCES}

1. Hogan P, Dall T, Nikolov P, American Diabetes Association. Economic costs of diabetes in the US in 2002. Diabetes Care 2003;26:917-932. 
2. Wagner EH, Sandhu N, Newton KM, McCulloch DK, Ramsey SD, Grothaus LC. Effect of improved glycemic control on health care costs and utilization. JAMA 2001;285:182-189.

3. Sidorov J, Shull R, Tomcavage J, Girolami S, Lawton $\mathrm{N}$, Harris R. Does diabetes disease management save money and improve outcomes? A report of simultaneous short-term savings and quality improvement associated with a health maintenance organizationsponsored disease management program among patients fulfilling health employer data and information set criteria. Diabetes Care 2002;25:684-689.

4. Menzin J, Langley-Hawthorne C, Friedman M, Boulanger L, Cavanaugh R. Potential short-term economic benefits of improved glycemic control: a managed care perspective. Diabetes Care 2001;24:51-55.

5. The Diabetes Control and Complications Trial Research Group. The effect of intensive treatment of diabetes on the development and progression of longterm complications in insulin-dependent diabetes mellitus. N Engl J Med 1993;329:977-986.

6. Gilmer TP, O'Connor PJ, Manning WG, Rush WA. The cost to health plans of poor glycemic control. Diabetes Care 1997;20:1847-1853.

7. The American College of Endocrinology/The American Association of Clinical Endocrinologists. Implementation Conference for ACE Outpatient Diabetes Mellitus Consensus Conference Recommendations: position statement. February 2, 2005. Available at: www.aace.com/meetings/consensus/odimplementation/PositionStatement.pdf. Accessed March 14, 2006.
8. American Diabetes Association position statement: standards of medical care in diabetes. Diabetes Care 2006;19:S4-S42.

9. Mukhtar Q, Jack LJ Jr., Martin M, Murphy D, Rivera M. Evaluating progress toward Healthy People 2010 national diabetes objectives. Prev Chronic Dis 2006;3:A11.

10. Sidorov J, Gabbay R, Harris R, et al. Disease management for diabetes mellitus: impact on hemoglobin A1c. Am J Manag Care 2000;6:1217-1226.

11. Orr P, Hamar B, Carr D, et al. The impact of telephonic intervention on compliance with LDL and A1c testing in a disease management program. Circulation 2006;113:788-834.

12. Harwell TS, Dettori N, McDowall JM, et al. Do persons with diabetes know their A1c number? Diabetes Educ 2002;28:99-105.

13. Fowles JB, Rosheim K, Fowler EJ, Craft C, Arrichiello L. The validity of self-reported diabetes quality of care measures. Int J Qual Health Care 1999;11:407-412.

Address reprint requests to: Patty M. Orr, M.S.N., Ed.D., R.N. Healthways, Inc.

3841 Green Hills Village Dr. Ste. 300 Nashoille, TN 37215

E-mail: patty.orr@healthways.com 